\title{
Perioperative Anesthetic Management of a Case of Rare Ectopic Pheochromocytoma
}

\author{
Aihua Jiang ${ }^{\text {a, c }}$, Haiqiao Zhang ${ }^{\text {a, c }}$, Xiaofei Liu ${ }^{\text {a }}$, Huishan Zhao ${ }^{\text {b, d }}$
}

\begin{abstract}
We report an unusual case of pheochromacytoma and investigate the perioperative anesthetic management methods for giant abdominal aortic pheochromocytoma. We analyzed and summarized the recent clinical anesthetic management and experience in the hospital for a giant abdominal aortic pheochromocytoma, with the size of $20 \times 14 \times$ $5 \mathrm{~cm}$. Under general anesthesia combined with TAPB, the resection of the giant abdominal aorta pheochromocytoma was successfully completed, and the patient was cured and discharged. Well planned and careful management resulted in patient with stable blood pressure, blood volume and cardiac functions, free from complications. We concluded that, as for the patient in this case, the clear preoperative pathological diagnosis, intraoperative joint application of a variety of vasoactive drugs, the long time usage of postoperative high dose of norepinephrine, as well as effective analgesic measures, were the focus of the patient's perioperative anesthesia management, providing protection to the prognosis and turnover to the patients.
\end{abstract}

Keywords: Pheochromocytoma; Perioperative period; Anesthesia management

\section{Introduction}

Ectopic pheochromocytoma refers to extra-adrenal pheochromocytoma, and is relatively rare in clinical settings. Overall, ectopic pheochromocytoma accounts for $10-15 \%$ of all the pheochromocytomas $[1,2]$. It is more commonly seen in the abdominal aorta, near the renal hilus, and inferior vena cava $[3,4]$. Excess endogenous catecholamine secretion is the basic pathophysiology change of pheochromocytoma. Clinically, the most common symptom is hypertension, often accompa-

\footnotetext{
Manuscript submitted October 30, 2017, accepted November 23, 2017

aDepartment of Anesthesia, the Affiliated Yantai Yuhuangding Hospital of Qingdao University, Yantai, China

${ }^{b}$ Central Laboratory, the Affiliated Yantai Yuhuangding Hospital of Qingdao University, Yantai, China

${ }^{\mathrm{c}}$ These authors contributed equally to this paper.

${ }^{\mathrm{d} C}$ Corresponding Author: Huishan Zhao, Central Laboratory, the Affiliated Yantai Yuhuangding Hospital of Qingdao University, Yantai 264000, China. Email: zhaohuishan1011@163.com
}

doi: https://doi.org/10.14740/wjon1065e nied by headache, palpitations, sweaty palms, anxiety, etc. [2, 5]. Due to the ectopic nature and lack of specific symptoms and that many factors can induce catecholamine crisis, ectopic pheochromocytoma is easily missed preoperatively. The lack of preoperative diagnosis and some of severe conditions associated with pheochromocytoma in patients with pheochromocytoma who had no appropriate preoperative preparations results in the perioperative mortality as high as $47 \%$ [1]. Thus, preoperative diagnosis, sufficient preoperative preparations, and alert of the complications associated with the condition are highly essential in surgically managing the condition. In this paper, we analyzed and summarized retrospectively the clinical data of one case of a large abdominal aortic ectopic pheochromocytoma, $20 \times 14 \times 5 \mathrm{~cm}$ in size, recently treated in our hospital. The preoperative clinical symptoms were not typical; the intraoperative blood pressure fluctuated dramatically, with sustained postoperative hypotension. The paper summarized the perioperative anesthesia management in order to provide reference to managing similar cases for reducing the risk.

\section{Case Report}

\section{Clinical information}

The patient was a middle-aged man, 45 years old, weighing 61 $\mathrm{kg}$. The mass was found when the patient underwent a physical check-up 1 year ago. The mass had no tenderness, no abdominal pain or abdominal distension. No headache, dizziness, palpitations, fever or other discomfort, and self-sensing sweating were reported. No treatment was given at this stage. Recently, the patient felt that the lump gradually increased in size, and was admitted to hospital for additional examination. The anamnesis was hypertension for half a year, with the maximum blood pressure up to $180 / 110 \mathrm{~mm} \mathrm{Hg}$ and no regular intake of antihypertensive drugs. Physical examination showed body temperature $(\mathrm{T}) 36.4{ }^{\circ} \mathrm{C}$, heart rate $(\mathrm{P}) 62$ times $/ \mathrm{min}$, respiratory rate (R) 18 times/min, and blood pressure (bp) $154 / 98 \mathrm{~mm}$ $\mathrm{Hg}$. The abdomen was bulging. A lump was palpable, with the diameter of about $20 \mathrm{~cm}$ in the left abdomen. Biochemically test results showed urine vanilla mandelic acid (VMA) 528.69 $\mu \mathrm{mol} / 24 \mathrm{~h}$ (normal range: 15.66 - 85.88), urine methoxylated norepinephrine in total $1,957.65 \mu \mathrm{g} / 24 \mathrm{~h}$ (normal range: 119 - 451), normal urinary methoxyprogesterine in total, normal blood cortisols (at 0:00, 8:00 am, and 16:00 pm), and the normal rest test items. In terms of clinical imaging result, the abdominal CT [6] showed a lump with the size of about $11.4 \mathrm{~cm}$ 


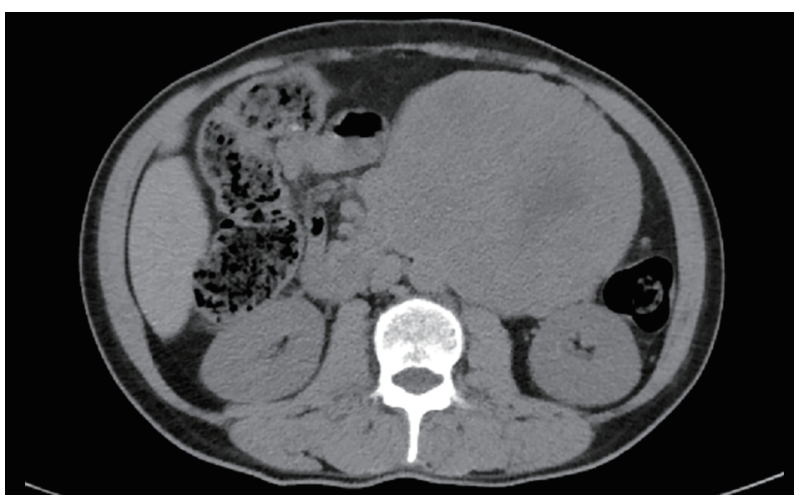

Figure 1. Abdominal CT scan showing left abdominal mass.

(AP), $110.9 \mathrm{~cm}(\mathrm{RL})$, and $15.8 \mathrm{~cm}$ (SI) in the left side of the abdominal cavity (Fig. 1); cardiac color Doppler showed left ventricular hypertrophy, and left ventricular diastolic dysfunction EF 72\%; chest CT showed double emphysema and lung bulb; ECG and lung function were normal.

\section{Methods}

\section{Preoperative preparations}

To make firm diagnosis, a decision was made to carry out retroperitoneal mass biopsy with ultrasound guidance under monitored anesthesia care (MAC). The punch biopsy was carried out successfully. During the procedure, the maximum blood pressure was up to $178 / 101 \mathrm{~mm} \mathrm{Hg}$ and patient felt no discomfort. Pathological examination showed the following: morphologically consistent with pheochromocytoma, immunohistochemically cytokeratin (-), CgA (+), Syn (+), S-100 $(+)$, and Ki-67 (+ about 1\%) (Fig. 2). Through multidisciplinary MDT (Department of Vascular Surgery, Anesthesiology, Urology, Ultrasound Intervention, Infectious Diseases, Endocrinology, Intensive Care Unit), a collective diagnosis of ectopic (abdominal aorta) pheochromocytoma was made. The patient was given persral administration of phenoxybenzamine $10 \mathrm{mg}$ bid, metoprolol tablets $25 \mathrm{mg}$ bid, nifedipine controlled release tablets $32 \mathrm{mg}$ qd, 3 weeks before the surgery until to the day of surgery. Six days prior to surgery, in addition to normal diet, the patient received $1,000 \mathrm{~mL}$ sodium lactate Ringer solution, $500 \mathrm{~mL}$ converted sugar sodium chloride solution for expansion per day. Patient's blood pressure was contributed at $135-110$ and $75-90 \mathrm{~mm} \mathrm{Hg}$.

\section{Intraoperative anesthesia management}

In the operating room, patient was well prepared with ECG heart rate at 75 times/min, oxygen saturation at $99 \%$, and noninvasive right upper limb blood pressure at 145/85 $\mathrm{mm} \mathrm{Hg}$. Patient was given continuously suprofen at $5 \mu \mathrm{g}$ and romethorphan $0.5 \mu \mathrm{g} / \mathrm{kg} / \mathrm{min}$ for $10 \mathrm{~min}$, and then changed to maintenance dose of $0.2 \mu \mathrm{g} / \mathrm{kg} / \mathrm{h}$. After the patient fell asleep, radial

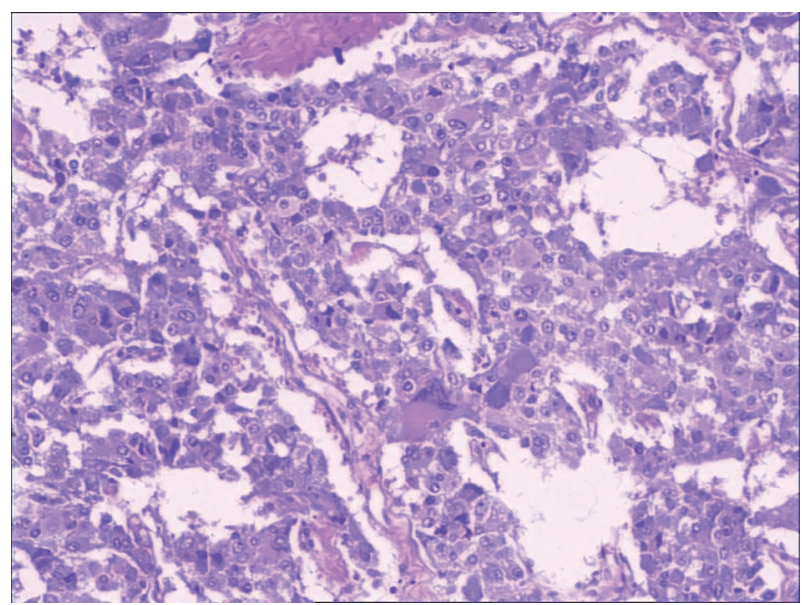

Figure 2. Eccentric nuclei large cell is shown by basophil included. Immunohistochemistry shows CgA (+), Syn (+).

artery cannulation and subclavian vein cannulation were established to allow monitoring invasively blood pressure and central venous pressure (CVP). Muscle relaxation and anesthesia depth were also continuously monitored. At this stage, $4 \mathrm{mg}$ of midazolam, $30 \mu \mathrm{g}$ of sufentanil, $150 \mathrm{mg}$ of propofol, and $50 \mathrm{mg}$ of rocuronium bromide were induced. After the muscle relaxation and anesthesia depth met the requirements of intubation, tracheal intubation was carried out followed by assisted ventilation. The invasive radial artery pressure rose sharply to 200/102 $\mathrm{mm} \mathrm{Hg}$ and the heart rate rose to 118 beats/min during intubation (Fig. 3); bolus injected $2 \mathrm{mg}$ of phentolamine and $30 \mathrm{mg}$ of esmolol were given in response. During the surgical procedure, $4 \mathrm{mg} / \mathrm{kg} / \mathrm{h}$ of propofol, $0.15 \mu \mathrm{g} / \mathrm{kg} / \mathrm{min}$ of remifentanil, and sevoflurane 1 - 2 MAC were continuously given to maintain BIS value within the range of 40 - 50; rocuronium was also intermittently injected to make TOF $<1$. During the surgical exploration, the blood pressure rose sharply to $192 / 120$ $\mathrm{mm} \mathrm{Hg}$, and heart rate increased to 118 times/min upon touch the tumor (Fig. 3). Exploration was temporarily halted in order to adjust the blood pressure to $160 / 85 \mathrm{~mm} \mathrm{Hg}$, heart rate of $110 \mathrm{times} / \mathrm{min}$ or less with the following procedure: bolus injection of $2 \mathrm{mg}$ of phentolamine and $30 \mathrm{mg}$ of esmolol, then continuously infusion, via pump, of phentolamine at the rate of $0.5 \mathrm{mg} / \mathrm{min}$ via pump, sodium nitroprusside at the rate of $1 \mu \mathrm{g} / \mathrm{kg} / \mathrm{min}$, and esmolol at the rate of $100 \mu \mathrm{g} / \mathrm{kg} / \mathrm{min}$. Surgical procedure went ahead when this was reached during which blood pressure was maintained at 150 - 195/90 - 102 mm Hg (Fig. 3). During operation, the reflux vein on the tumor surface was sutured. It was found that the inferior mesentery artery and the inferior mesentery vein were intimately conjoined the edge of the tumor and could not be dissected away from the tumor body. Two branches of arteries derived from aorta fed the tumor. These vessels, namely inferior mesentery artery and vein and the feeding aortic branches, were all ligated and cut away from the tumor body, to allow completely removal of the tumor.

Three minutes before removing the tumor, antihypertensive drugs (to maintain the BIS value within 50 - 60) were stopped and fluid infusion was accelerated to maintain the 


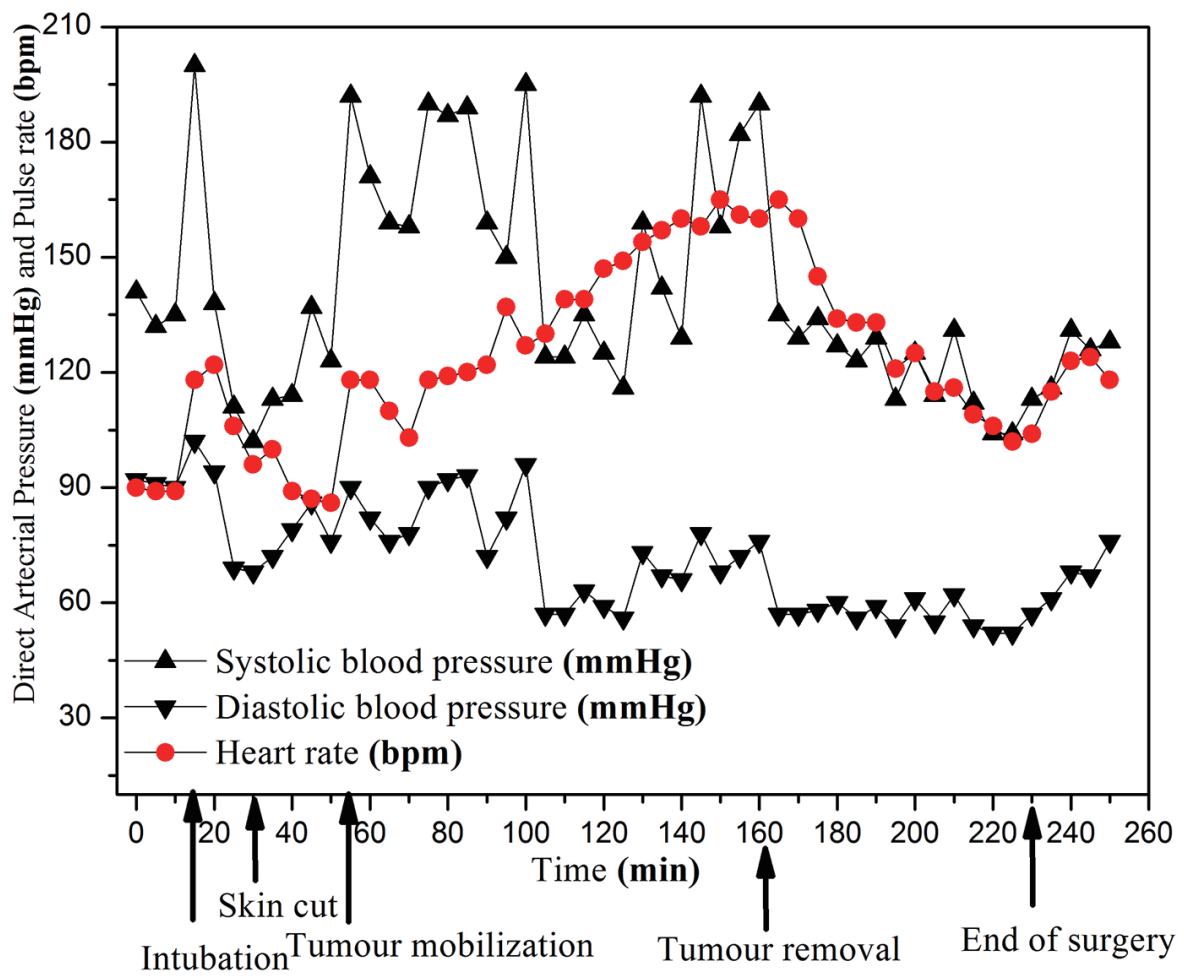

Figure 3. Invasive arterial blood pressure and heart rate curves.

CVP at $8-12 \mathrm{~cm} \mathrm{H}_{2} \mathrm{O}$. After resection of the tumor, the patient's blood pressure dropped to $115 / 57 \mathrm{~mm} \mathrm{Hg}$ (Fig. 3) and in response $0.2 \mathrm{mg}$ of norepinephrine was given followed by continuous administration at the rate of $0.5 \mu \mathrm{g} / \mathrm{kg} / \mathrm{min}$ to maintain blood pressure within 104 - 135/52 - 76 mm Hg (Fig. 3). In total, the surgery lasted for $3 \mathrm{~h} 20 \mathrm{~min}$, with 1,200 mL of blood loss, and $400 \mathrm{~mL}$ of autologous blood recovery. After the completion of the surgery, norepinephrine was maintained at 1 $\mu \mathrm{g} / \mathrm{kg} / \mathrm{min}$. When the patient exited the operating room, heart rate $(\mathrm{P})$ was 118 times/min, respiratory rate $(\mathrm{R})$ was 12 times/ min and the blood pressure (bp) was 128/76 mm Hg (Fig. 3).

\section{Postoperative management}

Postoperatively, analgesia used was sufentanil, given at 2 $\mathrm{mL} / \mathrm{h}(150 \mu \mathrm{g} / 100 \mathrm{~mL})$. The tracheal tube was removed $4 \mathrm{~h}$ postoperatively. Norepinephrine was given continuously $(0.15$ - $0.5 \mu \mathrm{g} / \mathrm{kg} / \mathrm{min}$ ), to maintain blood pressure at 110 - 130/55 $70 \mathrm{~mm} \mathrm{Hg}$. On the postoperative fifth day, all the vital signs were stable, and at this point the patient returned to the general ward for subsequent treatment.

\section{Postoperative monitor and pathological results}

The visual analogue scale (VAS) results were less than 4 at $6,12,24$ and $48 \mathrm{~h}$ after the surgery and the analgesia effect was perfect. The total amount of methotrexadenadine received was $992.20 \mu \mathrm{g} / 24 \mathrm{~h}$ on the postoperative seventh day, signifi- cantly lower than pre-operation amount, although remaining higher than normal level. This was likely due to the continuous administration of norepinephrine after surgery. VMA, liver and kidney function tests were normal and the blood pressure maintained at 122/85 $\mathrm{mm} \mathrm{Hg}$ and heart rate at $68 \mathrm{times} / \mathrm{min}$.

Pathological examination revealed that the tumor was large in size, $20 \times 14 \times 5 \mathrm{~cm}$ (Fig. 4). The capsule was intact, part of which was affected by the tumor. A proportion of tumor cells showed signs of fast growing with nuclear mitosis at 3/50 HPF. The pathological diagnosis was pheochromocytoma; immunohistochemistry showed CgA (+), Syn (+), S-100 (+), Ki67 (+ about 1\%). The patient was cured and discharged on the 10 th day after surgery with no complications.

\section{Discussion}

The clinical manifestations of ectopic pheochromocytoma are in line with adrenal pheochromocytoma, most commonly seen in young adults [5]. Preoperative diagnosis of ectopic pheochromocytoma remains a challenge, due to lack of specific symptoms and awareness. In the present case report of abdominal aortic pheochromocytoma, the patients had a history of hypertension and significantly raised VMA of $528.69 \mu \mathrm{mol} / 24$ $\mathrm{h}$ (normal range: $15.66-85.88$ ) and total urinary methoxylated norepinephrine at 1,957.65 $\mu \mathrm{g} / 24 \mathrm{~h}$ (normal range: 119 - 451), thus raising initial suspicion of an ectopic pheochromocytoma. Ultrasound-guided biopsies facilitated the diagnosis. It has been reported that the mortality was only $0-3 \%$ for patients with adequate preoperative preparation; however, the mortali- 


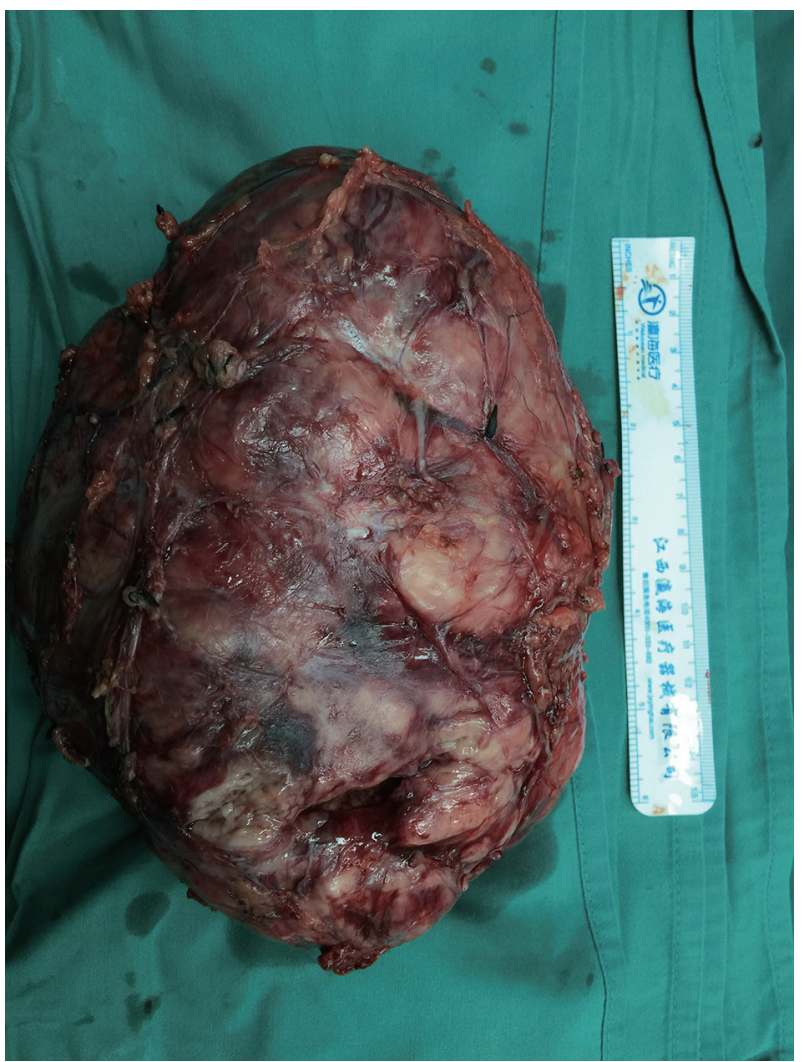

Figure 4. Resected ectopic pheochromocytoma.

ty rate would go up to $43 \%$ for patients without diagnosed quiescent pheochromocytoma $[7,8]$. Therefore, the preoperative diagnosis is one of the prerequisites for reducing the risk of perioperative anesthesia. After making the definite diagnosis, preoperative easing blood pressure, expansion of blood volume, this step is quite important in preoperative preparation stage, which even determines to a certain extent the postoperative outcome of pheochromocytoma [9].

It has been reported that, for pheochromocytoma patients, anesthesia induction, tracheal intubation, and whilst tumor being moved might induce release of a large amount of catecholamines $[10,11]$. Although preoperative preparation has been fully conducted in this case, the blood pressure and heart rate had been markedly raised for several times, after tracheal intubation under deep anesthesia, surgically exploring the tumor, as well as moving the tumor during the operation. We have relied on large doses of simultaneous administration of vasoactive drugs $(60 \mathrm{mg}$ of phentolamine, $35 \mathrm{mg}$ of sodium nitroprusside and $1,000 \mathrm{mg}$ of esmolol) and successfully managed this difficult condition. We believe that the reasons for blood pressure fluctuations and heart rate fluctuations for long duration have been the large size of the tumor and the particularly strong activity of catecholamines secretion $[12,13]$. One particular point with the present case is that after the resection, there was no sharp decline in blood pressure as one might fear. We strongly believe that the reason behind this success comes from the following three actions: first, injection of a large amount of liquid for expansion before surgery and resection; second, withdrawal of all antihypertensive drugs 3 min before completing resection of the tumor; third, immediate injection and pump of norepinephrine after resection of the tumor (10 $\mathrm{mg}$ of norepinephrine in the present case).

Postoperative hypotension has been one of the most common complications for pheochromocytoma patients, and in severe cases, this may lead to cardiac arrest $[13,14]$. When dealing with tumors with giant size and the strong function activity, in order to prevent lack of catecholamines and other substances after tumor removal (withdrawal), we adopted continued administration of norepinephrine $(120 \mathrm{mg})$ for 5 days postoperatively, as well as $3,800 \mathrm{~mL} /$ day of liquid therapy, which effectively avoided the occurrence of low blood pressure and cardiovascular events. In addition, the perfect postoperative multi-mode analgesia accelerated the rehabilitation of the patient.

The perioperative anesthesia management, from the preoperative ultrasound-guided retroperitoneal biopsy under MAC and MDT participated by anesthetists, to the active maintaining stability during surgery, and to the precise and postoperative analgesia, contemporary anesthesiology is essential in developing the perioperative medical evolution. But to retrospect the case, there remains room for improvement. First, although there was preoperative diagnosis of ectopic pheochromocytoma through guided biopsies, there is no more report about the safety of preoperative pathological diagnosis of the ectopic pheochromocytoma. Second, the case has been managed successfully, but due to the giant tumor size and the strong functionality of tumor cells, it was difficult to fully control blood pressure during the process of anesthesia, which led to blood pressure and heart rate fluctuations and unstable circulatory. Third, the present case report is unable to provide a long term follow-up of the patients. The literatures report the adrenal (ectopic) pheochromocytoma malignant ratio is high, about $15-35 \%$ [14]. The present reports tend to share the positive experience of managing a large and functionally active ectopic pheochromocytoma by anesthesia prior, during and after surgery. The case will be followed in long term, together with other cases.

In conclusion, giant and functional ectopic pheochromocytoma of aorta, a rare condition, is associated with clinical manifestations that reflect the function of the tumor cells, namely hypertension and tachycardia. The management of the patients during surgery requires carefully planning pre-, during and after surgery to control the blood pressure, blood volume and heart rate. This needs the participation of anesthetists of all stages of the medical care.

\section{References}

1. Tagaya N, Kasama K, Suzuki N, Taketsuka S, Horie K, Kubota K. Video-assisted bullectomy using needlescopic instruments for spontaneous pneumothorax. Surg Endosc. 2003;17(9):1486-1487.

2. Tu YR, Li X, Lin M, Lai FC, Li YP, Chen JF, Ye JG. Epidemiological survey of primary palmar hyperhidrosis in adolescent in Fuzhou of People's Republic of China. Eur J Cardiothorac Surg. 2007;31(4):737-739. 
3. Sahdev A, Sohaib A, Monson JP, Grossman AB, Chew SL, Reznek RH. CT and MR imaging of unusual locations of extra-adrenal paragangliomas (pheochromocytomas). Eur Radiol. 2005;15(1):85-92.

4. Ito MK, Talbert RL, Tsimikas S. Statin-associated pleiotropy: possible beneficial effects beyond cholesterol reduction. Pharmacotherapy. 2006;26(7 Pt 2):85S-97S; discussion 98S-101S; quiz 106S-108S.

5. Zhang $\mathrm{P}$, Hao GQ, Lin YB, et al. Giant abdominal nonfunctioning pheochromocytoma. Chinese Journal of Gastroenterology. 2008;13(4):252-253.

6. Wang DB, Zhang H, He GX, et al. CT diagnosis of ectopic pheochromocytoma. Chin J Radiol. 1998;(02):36-39.

7. Pan DL, Li HZ, Luo AL, et al. Diagnosis and treatment of pheochromocytoma: a fifty-year clinical experience. Chin J Urol. 2005:(11):6-8.

8. Zhang GF, CUI EF, Yao HX, et al. Clinical and CT diagnostic analysis of ectopic pheochromocytoma. China Practical Medical. 2011;6(15):43-44.
9. Zhuang Xiaofeng. Perioperative management of ectopic pheochromocytoma. Journal of Surgery Concepts \& Practice. 2012;17(04):328-331.

10. Treschan TA, Peters J. The vasopressin system: physiology and clinical strategies. Anesthesiology. 2006;105(3):599612; quiz 639-540.

11. Arora S, Vargo S, Lupetin AR. Computed tomography appearance of spontaneous adrenal hemorrhage in a pheochromocytoma. Clin Imaging. 2009;33(4):314-317.

12. Eisenhofer G, Rivers G, Rosas AL, Quezado Z, Manger WM, Pacak K. Adverse drug reactions in patients with phaeochromocytoma: incidence, prevention and management. Drug Saf. 2007;30(11):1031-1062.

13. Manger WM. An overview of pheochromocytoma: history, current concepts, vagaries, and diagnostic challenges. Ann N Y Acad Sci. 2006;1073:1-20.

14. Ilias I, Pacak K. Current approaches and recommended algorithm for the diagnostic localization of pheochromocytoma. J Clin Endocrinol Metab. 2004;89(2):479-491. 